OPTICA ACTA, 1971, VoL. 18, No. 12, 891-902

\title{
Application of speckling for in-plane vibration analysis
}

\author{
H. J. TIZIANI \\ Institute of Applied Physics of the Swiss Federal Institute of \\ Technology, (ETH), 8049 Zürich, Switzerland
}

(Received 26 fuly 1971)

\begin{abstract}
Time averaged as well as doubly exposed speckle patterns of in-plane moving objects are studied. These speckle patterns are recorded photographically; their Fourier transform shows interference fringes corresponding to the lateral displacement of the object. It leads to a very simple engineering tool for the analysis of mechanical vibrations as well as lateral displacements. Theoretical and experimental results will be shown.
\end{abstract}

\section{Introduction}

An optically rough surface illuminated with coherent light leads to a speckled appearance in the image space. The speckling and its applications for the detection of nodal lines for example was investigated recently by a number of workers [1-6]. Leendertz [7] suggested a method for measuring deformations in the image of the speckle pattern applying two oblique illuminating waves. Burch and Tokarski [8] showed that, by the registration of a speckle pattern on a photographic plate $m$ times with $m-1$ displacements of the plate, interference patterns are observed in the Fourier plane. A lateral displacement of the optically rough surface between exposures shifts the speckle pattern in the image plane, too. The Fourier transform of the doubly exposed speckle pattern, which may be registered on a photographic emulsion, reveals interference fringes in the Fourier plane which are a measure for the lateral displacement in the object plane. This was also found by other authors [9-11]. In the work to be reported, the speckling was analysed for the study of the mechanical oscillations of a tuning fork of a new electronic watch.

In the following analysis the intensity distribution of the time averaged as well as the laterally displaced speckling are analysed in the image plane. After development of the plate or film, the interference patterns in the Fourier plane are studied.

\section{Image of a laterally displaced rough object when illuminated coherently}

A plane wave at an angle specified by $\beta$ illuminates the object $A_{0}(\mathbf{u})$. To obtain a true Fourier transform on the entrance pupil sphere of the optical system a spherical wave converging to the entrance pupil of the optical system should be used [3], but this has no consequences on the results. The complex amplitude of the roughness of the surface at a point $P(u)$ in the object plane may be written as the superposition of a set of waves

$$
\sum_{p} \Psi\left(\mathbf{x}_{p}\right) \exp \left[i 2 \pi \mathbf{x}_{p} \cdot \mathbf{u}\right]
$$

which may be written as an integral in the limit to represent the distribution of 
complex amplitudes as a function of $\mathbf{u}$ by its Fourier integral. $\mathbf{u}$ and $\mathbf{x}_{p}$ are two-dimensional vectors, representing the coordinates $(u, v)$ in the object and $\left(x_{p}, y_{p}\right)$ in the pupil plane. According to customary usage [12]

$$
\left.\begin{array}{ll}
u=\frac{n \sin \alpha}{\lambda} \xi, & x_{p}=\frac{X_{p}}{h}, \\
v=\frac{n \sin \alpha}{\lambda} \eta, & y_{p}=\frac{Y_{p}}{h},
\end{array}\right\}
$$

with the notations shown in figure 1 (one-dimensional).

$\alpha, \alpha^{\prime}=$ aperture angle of the optical system in the object and image space respectively,

$n^{\prime}, n^{\prime}=$ refractive index in the object and image space,

$\lambda \quad=$ wavelength in vacuum,

$\xi, \eta=$ rectangular coordinates in the object space,

$x_{p}, y_{p}=$ normalized pupil coordinates,

$x, y=$ normalized coordinates of the intersection $X, Y$ of the finite rays with the pupil sphere,

$h, h^{\prime}=$ paraxial ray heights at the entrance and exit pupils respectively.

A similar notation will be used later for the exit pupil and image space.

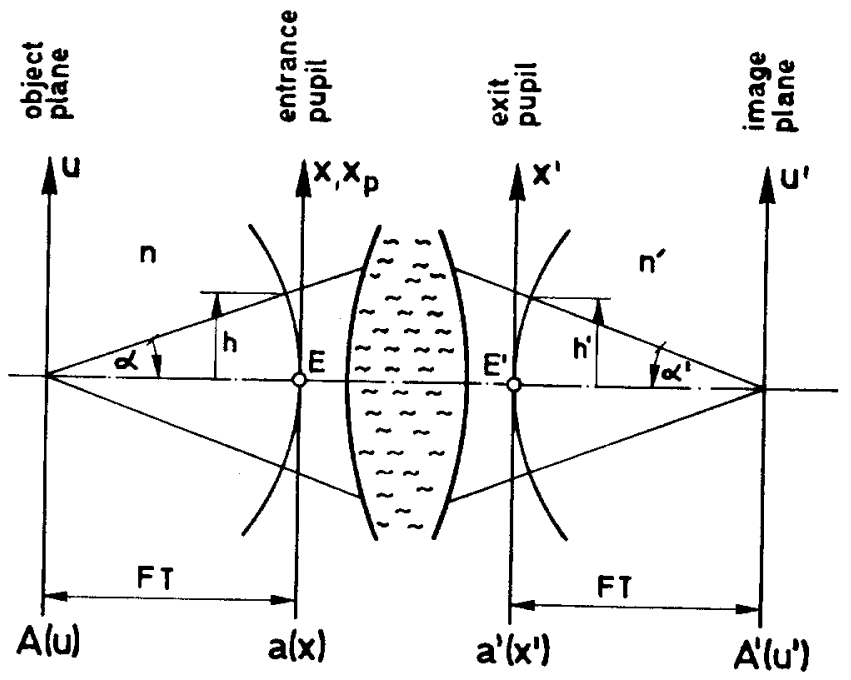

Figure 1. Image formation with coherent light.

The total complex amplitude in the object space can therefore be written as

$$
A(\mathbf{u})=A_{0}(\mathbf{u}) \exp \left[i 2 \pi \frac{\beta . \mathbf{u}}{\sin \alpha}\right] \sum_{p} \Psi\left(\mathbf{x}_{p}\right) \exp \left[i 2 \pi \mathbf{u} . \mathbf{x}_{p}\right] .
$$

The lateral displacement of the object (or part of it) may be $\Delta \mathbf{u}$. In that case the co mplex amplitude in the object space can be written as

$$
A(\mathbf{u}, \Delta \mathbf{u})=A_{0}(\mathbf{u}+\Delta \mathbf{u}) \sum_{p} \exp \left[i 2 \pi(\mathbf{u}+\Delta \mathbf{u})\left(\mathbf{x}_{p}+\frac{\beta}{\sin \alpha}\right)\right] \Psi\left(\mathbf{x}_{p}\right)
$$


and the distribution on the entrance pupil of the optical system

$$
a(\mathbf{x})=\int A(\mathbf{u}, \Delta \mathbf{u}) \exp [-i 2 \pi \mathbf{u} \cdot \mathbf{x}] d \mathbf{u}
$$

with the integration over the object space, leading to

$$
a(\mathbf{x})=\sum_{p} \Psi\left(\mathbf{x}_{p}\right) a_{0}\left(\mathbf{x}-\mathbf{x}_{p}-\frac{\beta}{\sin \alpha}\right) \exp [i 2 \pi \mathbf{x} . \Delta \mathbf{u}],
$$

where $a_{0}(\mathbf{x})$ is the spectrum of $A_{0}(\mathbf{u})$.

For a reasonable optical system $\mathbf{x}=\mathbf{x}^{\prime}$, where $\mathbf{x}, \mathbf{x}^{\prime}$ are the normalized entrance and exit pupil coordinates [12] (figure 1).

Considering an aberration-free system we obtain in the image space a complex amplitude

$$
A^{\prime}\left(\mathbf{u}^{\prime}, \Delta \mathbf{u}\right)=\int a(\mathbf{x}) \exp \left[i 2 \pi \mathbf{u}^{\prime} \cdot \mathbf{x}\right] d \mathbf{x}
$$

with the integration over the exit pupil of the optical system $\left[\sqrt{ }\left(x^{2}+y^{2}\right) \leqslant 1\right]$, giving

$$
A^{\prime}\left(\mathbf{u}^{\prime}, \Delta \mathbf{u}\right)=\sum_{p} \Psi\left(\mathbf{x}_{p}\right) A_{0}^{\prime}\left(\mathbf{u}^{\prime}+\Delta \mathbf{u}\right) \exp \left[i 2 \pi\left(\mathbf{u}^{\prime}+\Delta \mathbf{u}\right)\left(\mathbf{x}_{p}+\frac{\beta}{\sin \alpha}\right)\right],
$$

where $A_{0}^{\prime}\left(\mathbf{u}^{\prime}\right)$ is the image of $A_{0}(\mathbf{u})$. Since $\mathbf{u}^{\prime}$ and $\mathbf{u}$ are normalized in the same way and $n \xi \sin \alpha=n^{\prime} \xi^{\prime} \sin \alpha^{\prime}$ (sine condition), we can write for $\Delta \mathbf{u}=\Delta \mathbf{u}^{\prime}$. The intensity is given by

giving

$$
I\left(\mathbf{u}^{\prime}, \Delta \mathbf{u}^{\prime}\right)=\left|A^{\prime}\left(\mathbf{u}^{\prime}, \Delta \mathbf{u}^{\prime}\right)\right|^{2}
$$

$$
I\left(\mathbf{u}^{\prime}, \Delta \mathbf{u}^{\prime}\right)=\left|A^{\prime}{ }_{0}\left(\mathbf{u}^{\prime}+\Delta \mathbf{u}^{\prime}\right)\right|^{2} \sum_{p} \sum_{q} \Psi\left(\mathbf{x}_{p}\right) \Psi^{*}\left(\mathbf{x}_{q}\right) \exp \left[i 2 \pi\left(\mathbf{u}^{\prime}+\Delta \mathbf{u}^{\prime}\right)\left(\mathbf{x}_{p}-\mathbf{x}_{q}\right)\right] .
$$

In the following, the intensity distribution in the image and the Fourier plane of an optically rough object will be studied for the oscillating and displaced object.

3. Analysis of the time averaged in-plane movement in the image and the Fourier plane Considering the oscillating object with

$$
\Delta \mathbf{u}=\rho \cos 2 \pi \nu t
$$

with $\rho$ the amplitude of the lateral oscillation in the object space and $\nu$ the frequency. The expression (6) can be written as

$$
\begin{aligned}
I\left(\mathbf{u}^{\prime}, \rho^{\prime}, t\right)=\mid A^{\prime}{ }_{0}\left(\mathbf{u}^{\prime}+\left.\rho^{\prime} \cos (2 \pi \nu t)\right|^{2} \sum_{p} \sum_{q} \Psi\left(\mathbf{x}_{p}\right) \Psi^{*}\left(\mathbf{x}_{q}\right)\right. \\
\quad \times \exp \left[i 2 \pi \mathbf{u}^{\prime}\left(\mathbf{x}_{p}-\mathbf{x}_{q}\right)\right] \exp \left[i 2 \pi \rho^{\prime}\left(\mathbf{x}_{p}-\mathbf{x}_{q}\right) \cos 2 \pi \nu t\right] .
\end{aligned}
$$

The time averaged intensity is obtained by applying Jacobi's theorem and considering $\left|A^{\prime}{ }_{0}\left(\mathbf{u}^{\prime}+\rho^{\prime} \cos 2 \pi \nu t\right)\right|^{2} \approx\left|A^{\prime}{ }_{0}\left(\mathbf{u}^{\prime}\right)\right|^{2}$ for small $\left|\rho^{\prime}\right|$ 's and $t_{0} \gg 1 / \nu\left(t_{0}\right.$ is the response time of a typical detector, such as a photographic plate),

$$
\begin{array}{r}
\left\langle I\left(\mathbf{u}^{\prime}, \rho^{\prime}, t\right)\right\rangle=\left|A_{0}\left(\mathbf{u}^{\prime}\right)\right|^{2} \sum_{p} \sum_{q} \Psi\left(\mathbf{x}_{p}\right) \Psi^{*}\left(\mathbf{x}_{q}\right) J_{0}\left[2 \pi \rho^{\prime}\left(\mathbf{x}_{p}-\mathbf{x}_{q}\right)\right] \\
\times \exp \left[i 2 \pi \mathbf{u}^{\prime}\left(\mathbf{x}_{p}-\mathbf{x}_{q}\right)\right] .
\end{array}
$$


A further discussion of the time averaged speckling will follow in the Appendix.

The amplitude transparency of the developed photographic plate can approximately be written as [14]

$$
T\left(\mathbf{u}^{\prime}, \rho^{\prime}\right)=T_{0}-b t_{0}\left\langle I\left(\mathbf{u}^{\prime}, \rho^{\prime}, t\right)\right\rangle,
$$

where $t_{0}=$ exposure time, $T_{0}$ and $b$ are constants of the photographic material.

The developed photographic plate is now illuminated with a coherent wave. The complex amplitude in the Fourier plane can be written as

$$
a\left(\mathbf{x}_{1}\right)=\int T\left(\mathbf{u}^{\prime}, \rho^{\prime}\right) \exp \left[-i 2 \pi \mathbf{u}^{\prime} . \mathbf{x}_{1}\right] d \mathbf{u}^{\prime},
$$

where $\mathbf{x}_{1}=\mathbf{X}_{1} / \sin \alpha^{\prime} f_{1}$ and $\mathbf{X}_{1}$ stands for the rectangular coordinates $\left(X_{1}, Y_{1}\right)$ in the Fourier plane and $f_{1}$ is the focal length of the Fourier transforming lens.

For the analysis of an object where the amplitude as well as the direction of oscillation depend on the region of the object to be studied, an appropriate diaphragm can be used to select the region of almost constant displacement. The limits of the integral in the previous expression should therefore be from $\left|\mathbf{u}^{\prime}-\mathbf{u}_{0}^{\prime}\right| \leqslant r$, only ( $\mathbf{u}_{0}^{\prime}=$ position of the diaphragm of radius $r$ ).

To simplify the formula we ignore for $r \gg\left|\rho^{\prime}\right|$ the convolution with the Airy diffraction pattern which results from the finite size of the diaphragm. We neglect further the constant term due to the undiffracted light in expression (9). Hence, we obtain by substituting (8) and (9) in (10) and rearranging terms,

$$
a\left(\mathbf{x}_{1}\right) \approx\left[a_{0}\left(\mathbf{x}_{1}\right) \circledast a_{0}^{*}\left(-\mathbf{x}_{1}\right)\right] \circledast \int \Psi\left(\mathbf{x}_{1}+\mathbf{x}_{q}\right) \Psi^{*}\left(\mathbf{x}_{q}\right) d \mathbf{x}_{q} J_{0}\left[2 \pi \mathbf{x}_{1} \boldsymbol{\rho}^{\prime}\right],
$$

where $a\left(\mathbf{x}_{1}\right) \circledast a^{*}\left(-\mathbf{x}_{1}\right)$ represents the autocorrelation function of the spectrum of the image $A_{0}^{\prime}\left(\mathbf{u}^{\prime}\right)$. The integral $\int \Psi\left(\mathbf{x}_{1}+\mathbf{x}_{q}\right) \Psi^{*}\left(\mathbf{x}_{q}\right) d \mathbf{x}_{q}$ is the autocorrelation of the speckling in the pupil of the image-forming optical system. $\circledast$ indicates as customary the convolution.

If the object has a fine scale roughness the scattered light will lead to a uniform illumination of the pupil of the optical system and therefore the integration in the above expression can be extended over the exit pupil of the image-forming system [2].

If the autocorrelation of the spectrum of the image $A^{\prime}{ }_{0}\left(\mathbf{u}^{\prime}\right)$ is very narrow we can write the intensity in the Fourier plane as

$$
\left|a\left(\mathbf{x}_{1}\right)\right|^{2} \approx\left|\int \Psi\left(\mathbf{x}_{1}+\mathbf{x}_{q}\right) \Psi^{* *}\left(\mathbf{x}_{q}\right) d \mathbf{x}_{q}\right|^{2} J^{2}{ }_{0}\left[2 \pi \mathbf{x}_{1} \cdot \rho^{\prime}\right] .
$$

Ignoring the centre, there will be a gradual decrease of the autocorrelation function towards the edge of the exit pupil (figure 2). The Bessel function $J_{0}\left[2 \pi \rho . \mathbf{x}_{1}\right]$ is multiplied by the autocorrelation function of the speckling in the pupil, which leads to the fringes in figure 3. The results obtained by scanning the Fourier plane with a slit of width slightly larger than the speckle diameter determined by the diaphragm used to select the appropriate portion of constant movement of the image are shown in figures 2 and 3 . The slit was oriented parallel to the interference fringes in figure 3 and of width $0 \cdot 1 \mathrm{~mm}$. Figure $2(a)$ shows the autocorrelation function for the stationary object and the image-forming system 


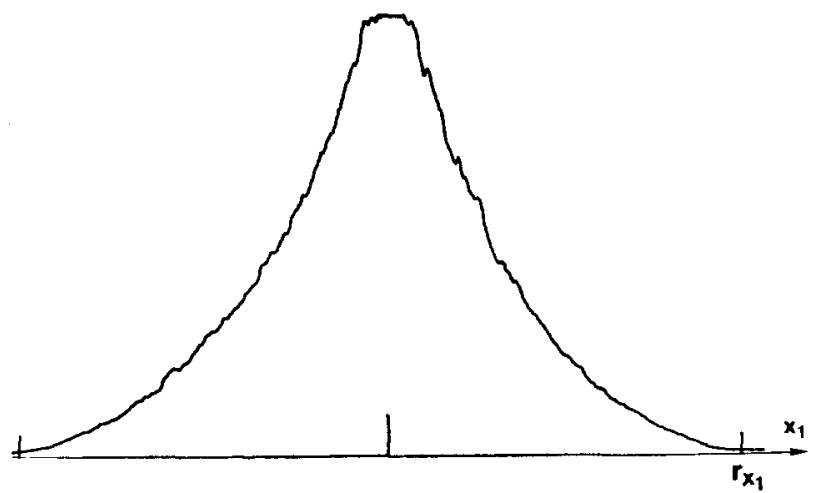

(a)

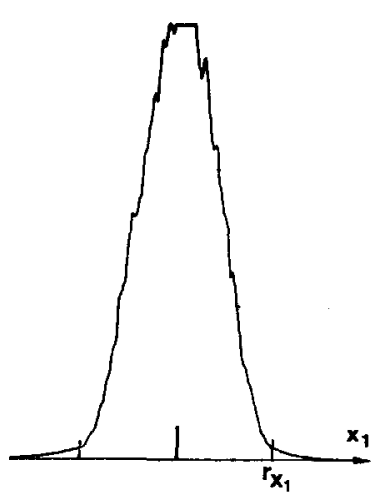

(b)

Figure 2. Autocorrelation function in the Fourier plane of the stationary object with the image-forming lens working at $(a) \mathrm{f} / 2 \cdot 8$ and $(b) \mathrm{f} / 11$.

working with a numerical aperture $\sin \alpha^{\prime}=0.045$ in figure $2(a)$ and 0.011 in figure $2(b)$. The corresponding intensity distributions in the Fourier plane for the vibrating object are shown in the figures $3(a)$ and $3(b)$ respectively. Some of the experimental details will be given in the next section. The radius $r_{x_{1}}$ where the autocorrelation function of the pupil approaches zero is given by $r_{x_{1}}=2 f_{1} \sin \alpha^{\prime}$, consequently, no fringes can be observed for $\left|\mathbf{x}_{1}\right|>r_{x_{1}}$. Since $r_{x_{1}}$ is directly proportional to the numerical aperture of the image-forming optical system in the image space (figure 2 ), $\sin \alpha^{\prime}$ should be large (high F-ratio) to obtain a number of fringes in the Fourier plane.

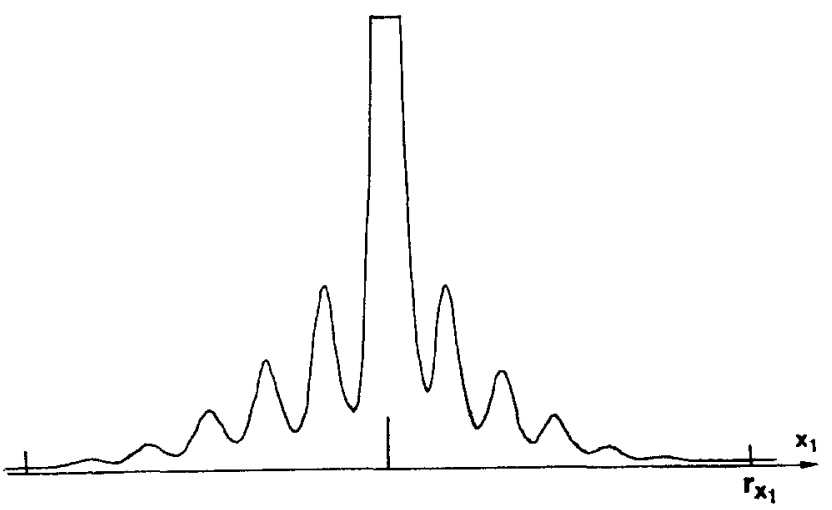

(a)

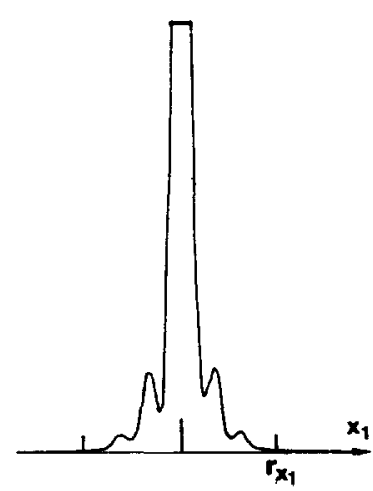

(b)

Figure 3. Intensity distribution in the Fourier plane for the oscillating object (amplitude $\left.2\left|\rho_{0}\right|=19 \mu \mathrm{m}\right)$ and the image-forming lens working at $(a) f / 2 \cdot 8$ and $(b) f / 11$.

From expression (12) we obtain the minima of intensity for $2 \pi \mathbf{x}_{1} \rho^{\prime}=2 \cdot 40$, $5 \cdot 52, \ldots$ Thus, denoting the separation of the first minima by $\left|p_{1}\right|$ and remembering the normalized coordinates in (1), we deduce the amplitude of oscillation $\left|p_{0}\right|$ in the object

$$
\left|\boldsymbol{p}_{\mathbf{0}}\right|=0.76 \frac{f_{1} \lambda}{M\left|\mathbf{p}_{1}\right|}
$$


with $M$ as the lateral magnification of the imaging optical system and $f_{1}$ the focal length of the transforming lens. To improve the accuracy, higher-order minima of $J_{0}$ can be evaluated. It should be noted that the direction of the fringes is perpendicular to the lateral displacement in the object. Typical results are shown in figure 4 ; they could also be observed with white light.

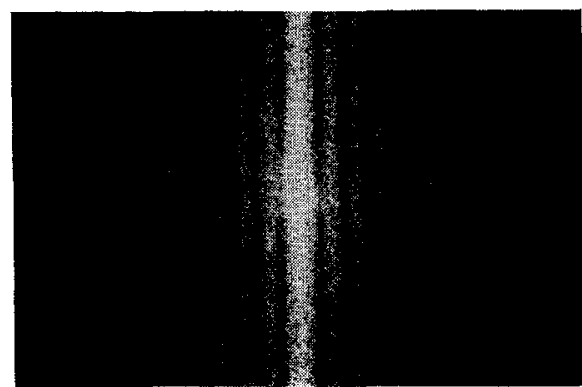

(a)

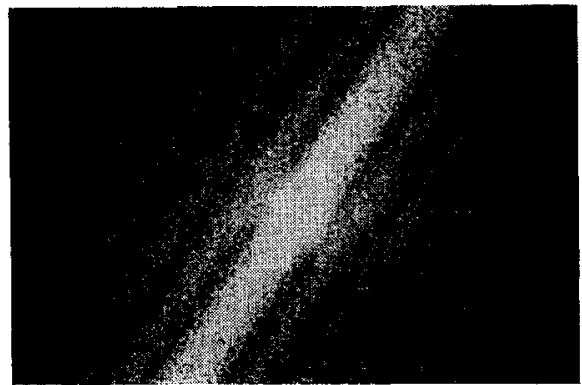

(b)

Figure 4. Photographs of the fringes in the Fourier plane of the time average speckle pattern alone (a) $2\left|\rho_{0}\right|=19 \mu \mathrm{m}$ and $(b) 2\left|\rho_{0}\right|=13.4 \mu \mathrm{m}$.

An interesting result was obtained by the analysis of a doubly exposed speckle pattern in the Fourier plane. The Fourier transform of the speckle pattern of the oscillating object (time average) superimposed on that of the stationary object (both recorded in the image space) leads to a slightly modified expression of (12), namely

$$
\left|b\left(\mathbf{x}_{1}\right)\right|^{2}=\left|\int \Psi\left(\mathbf{x}_{1}+\mathbf{x}_{q}\right) \Psi^{*}\left(\mathbf{x}_{q}\right) d \mathbf{x}_{q}\right|^{2} \mid\left(K+\left.J_{0}\left[2 \pi \rho^{\prime} \cdot \mathbf{x}_{1}\right]\right|^{2},\right.
$$

where $K$ is a constant depending on the exposure time of the stationary and oscillating speckle pattern. For $K \geqslant\left|K_{0}\right|\left(K_{0}=\right.$ amplitude of the first minimum of $J_{0}$ ) the minima of $\left|b\left(\mathbf{x}_{1}\right)\right|^{2}$ occur for $J_{0}^{\prime}\left(2 \pi \rho^{\prime} . \mathbf{x}_{1}\right)=0$ and $J^{\prime \prime}{ }_{0}>0$ (and no longer for $J_{0}=0$ ), namely for $2 \pi \rho^{\prime} . \mathbf{x}_{1}=3 \cdot 83,7 \cdot 02 \ldots$ A comparison of the parallel interference fringes in the Fourier plane of the doubly exposed (stationary and time average) speckle pattern is shown in figure 5 .

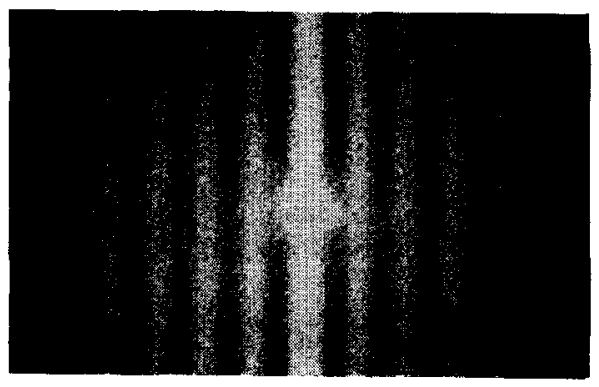

(a)

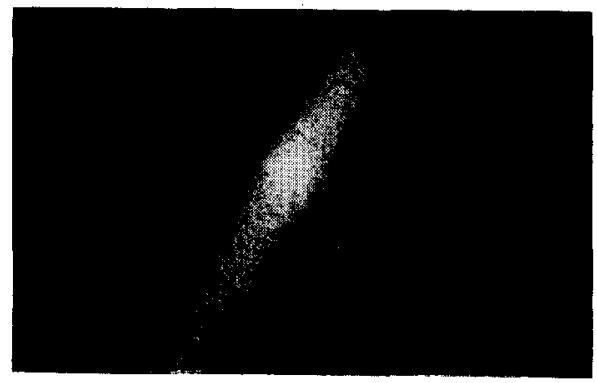

(b)

Figure 5. Photographs of the fringes in the Fourier plane of the time average speckle pattern superimposed on that of the stationary object (a) $2\left|\rho_{0}\right|=19 \mu \mathrm{m},\left(\right.$ b) $2\left|\rho_{0}\right|=$ $13 \cdot 4 \mu \mathrm{m}$. 
Different factors such as non-linearity of the photographic material and the influence of the diaphragm to select the portion of the object with approximately the same movement impair the contrast of the interference fringes slightly. By an appropriate choice of $K$ it was also possible to improve the contrast of the doubly exposed averaging method.

\section{Analysis of the in-plane displacement}

The lateral displacements of the speckling in the image will now be investigated in the Fourier plane. The movement of our oscillating object was reduced to a displacement by applying stroboscopic illumination, i.e. the two extreme positions are illuminated only [15]. Using the double exposure technique we obtain for the intensity distribution in the image of the displaced rough object the superposition of the contributions from each extreme position in turn and we can write

$$
I_{1}\left(\mathbf{u}^{\prime}, \Delta \mathbf{u}^{\prime}\right)=I\left(\mathbf{u}^{\prime}, \Delta \mathbf{u}^{\prime}\right)+I\left(\mathbf{u}^{\prime},-\Delta \mathbf{u}^{\prime}\right) .
$$

The two independent speckle patterns are shifted by $2 \Delta \mathbf{u}^{\prime}$ in the image space. As in the last section the complex amplitude in the Fourier plane can be written, by substituting the expressions (6) and (14) in (10), as

$$
a\left(\mathbf{x}_{1}\right)=\left[a_{0}\left(\mathbf{x}_{1}\right) \circledast a_{0}^{*}\left(-\mathbf{x}_{1}\right)\right] \circledast \int \Psi\left(\mathbf{x}_{1}+\mathbf{x}_{q}\right) \Psi^{*}\left(\mathbf{x}_{q}\right) d \mathbf{x}_{q} \cos \left(2 \pi \Delta \mathbf{u}^{\prime} . \mathbf{x}_{1}\right)
$$

The notation in the above expression is that used earlier.

The intensity distribution in the Fourier plane can be written by assuming, as previously, the autocorrelation of the spectrum of the image $A_{0}^{\prime}\left(\mathbf{u}^{\prime}\right)$ to be small,

$$
\left|a\left(\mathbf{x}_{1}\right)\right|^{2}=\left|\int \Psi\left(\mathbf{x}_{1}+\mathbf{x}_{q}\right) \Psi^{*}\left(\mathbf{x}_{q}\right) d \mathbf{x}_{q}\right|^{2} \cos ^{2}\left(2 \pi \mathbf{x}_{1} \cdot \Delta \mathbf{u}^{\prime}\right) .
$$

We obtain therefore cosine fringes in the Fourier plane of the doubly exposed speckle pattern multiplied with the square of the autocorrelation function of the speckling in the pupil. The other terms are those explained in expression (11). $\Delta \mathbf{u}^{\prime}$ is the displacement in the image space in the appropriate coordinates. Typical results are shown in figure 6. For a fringe-spacing $|\mathbf{p}|$ in the Fourier

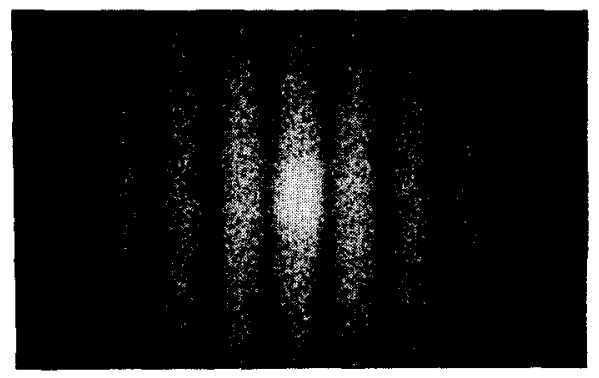

(a)

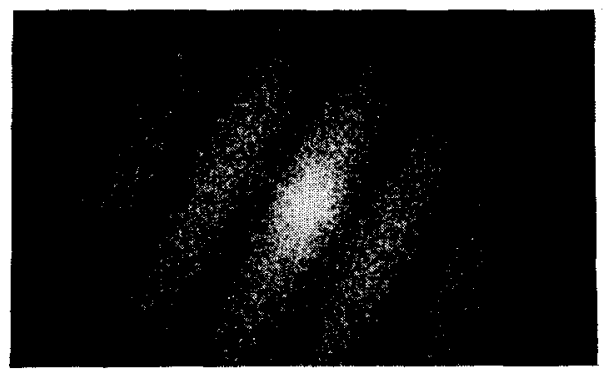

(b)

Figure 6. Photographs of the cosine fringes in the Fourier plane of the doubly exposed speckle pattern obtained with stroboscopic illumination of the tuning fork. (a) $2|\Delta \xi|=19 \mu \mathrm{m}$, (b) $2|\Delta \xi|=13.4 \mu \mathrm{m}$. 
plane we deduce the corresponding lateral displacement $|2 \Delta \xi|$ in the object (peak/peak) by

$$
2|\Delta \xi|=\frac{\lambda f_{1}}{M|\mathbf{p}|}
$$

\section{Experimental procedure}

The investigation was carried out basically for the vibration analysis of the tuning fork of a new electronic watch. The object was illuminated obliquely with a plane wave of a $\mathrm{He}-\mathrm{Ne}$ laser $\lambda=633 \mathrm{~nm}$ and imaged by an $\mathrm{F} / 2 \cdot 8$ lens with lateral magnification $M=-3$. The photographic material used was mostly Agfa-Gevaert Scientia 10 E70 for the registration of the speckled images and exposure times of a few seconds were necessary. Other, conventional photographic emulsions could be used for the registration of the speckling and no special care is needed for the processing to obtain the fringes in the Fourier plane with good contrast. The resonant frequency of the tuning fork was 1050 c.p.s. The amplitude of oscillation as well as its direction were considerably varying over the tuning fork and an appropriate diaphragm was chosen for the analysis of the photographic plates to select regions of approximately constant movements.

Figure 2 shows basically the square of the autocorrelation function of the exit pupil of the image-forming system. The point where the autocorrelation function is almost zero is marked with $r_{x_{1}}$ and agrees well with the theoretical results. The F-ratio of the image-forming system was $f / 2 \cdot 8$ for figure $2(a)$ and f/11 for figure $2(b)$. Figure $3(a), 3(b)$ show the time average interference fringes superimposed on the autocorrelation function $\left(2\left|\rho_{0}\right|=19 \mu \mathrm{m}\right)$.

Two regions of the tuning fork were selected for the analysis of the oscillations in the Fourier plane and the results are shown in figures $4(a)$ and $4(b)$ for the time average method and $5(a)$ and $5(b)$ for the doubly exposed speckle pattern, where the time average speckle pattern was superimposed on that of the stationary fork. It has been noted that the minima in figure 5 do not occur for $J_{0}=0$ as in figure 4 , but for $J_{0}^{\prime}=0$ and $J^{\prime \prime}{ }_{0}>0$ for $K \geqslant K_{0}$. By contrast, however, the doubly exposed speckle pattern of the extreme positions of the tuning fork when stroboscopic illumination is applied, leads to cosine fringes superimposed on the square of the autocorrelation of the speckling (over the exit pupil of the imageforming system) as shown in figures $6(a)$ and $6(b)$. The amplitude of oscillation for the regions chosen, shown in figures 4,5 and 6 , were $2\left|\rho_{0}\right|=19 \mu \mathrm{m}$ and $2\left|\rho_{0}\right|=13.4 \mu \mathrm{m}$ respectively. The results agree well with those found holographically [15].

\section{Conclusion}

The application of the speckling for the vibration analysis as well as for the measurements of lateral displacements and deformations is very promising. Displacements of the order of micrometers up to $0.2 \mathrm{~mm}$ were measured with this method. No reference wave is needed and the practical implementations of the analysis are not very demanding. The observation of the interference fringes as well as its interpretation is very easy. No special photographic materials and processing and no sophisticated equipment is necessary to perform the practical investigations and the method seems therefore to be an interesting tool for engineering purposes. 
In addition, the method applies for lateral movements in the presence of small tilts and movements perpendicular to the object plane. A movement out of plane reduces the contrast of the interference fringes.

\section{Appendix}

Investigations of the speckling in the image of an oscillating optically rough object

The speckling in the image of the oscillating optically rough surface will now be discussed briefly. Writing the summation in (8) as an integral in the limit and by considering the one-dimensional case we obtain by substituting for $x_{p}-x_{q}=x_{r}$ :

$$
\begin{aligned}
&\left\langle I\left(u^{\prime}, \rho^{\prime}, t\right)\right\rangle=\left|A^{\prime}{ }_{0}\left(u^{\prime}\right)\right|^{2} \iint \Psi\left(x_{r}+x_{q}\right) \Psi^{* *}\left(x_{q}\right) J_{0}\left[2 \pi \rho^{\prime} x_{r}\right] \\
& \times \exp \left[i 2 \pi u^{\prime} x_{r}\right] d x_{r} d x_{q},
\end{aligned}
$$

where $\left|A_{0}^{\prime}\left(u^{\prime}\right)\right|^{2}$ is the intensity of the image of the object $A_{0}(u)$. Noting that with the integral in expression (18) the Fourier transform of $\left(\Psi^{*} \circledast \Psi^{*}\right) J_{0}$ appears and we can write $\dagger$

$$
\left\langle I\left(u^{\prime}, \rho^{\prime}, t\right)\right\rangle=\left|A_{0}^{\prime}\left(u^{\prime}\right)\right|{ }^{2}\left|\tilde{\Psi}\left(u^{\prime}\right)\right|^{2} \circledast \frac{\operatorname{rect}\left(u^{\prime} \mid \rho^{\prime}\right)}{\pi \sqrt{ }\left(\rho^{\prime 2}-u^{\prime 2}\right)},
$$

where $\left|\tilde{\Psi}\left(u^{\prime}\right)\right|^{2}$ may be looked at as the power spectrum of the speckling in the image, where $\tilde{\Psi}\left(u^{\prime}\right)=\mathscr{F}\left[\Psi\left(x_{q}\right)\right]$.

For $\rho^{\prime}=0$ we obtain from expression (8) an image with speckling, namely : the superposition of randomly phased waves from the exit pupil and its shape and size determines the predominant shape and size of the speckles [1-3].

$I\left(u^{\prime}\right)=\left|A_{0}^{\prime}\left(u^{\prime}\right)\right|^{2} \sum_{p} \sum_{q}\left|\Psi\left(x_{p}\right)\right| \Psi^{*}\left(x_{q}\right) \mid \cos \left[2 \pi u^{\prime}\left(x_{p}-x_{q}\right)+\arg \Psi\left(x_{p}\right)-\arg \Psi\left(x_{q}\right)\right]$.

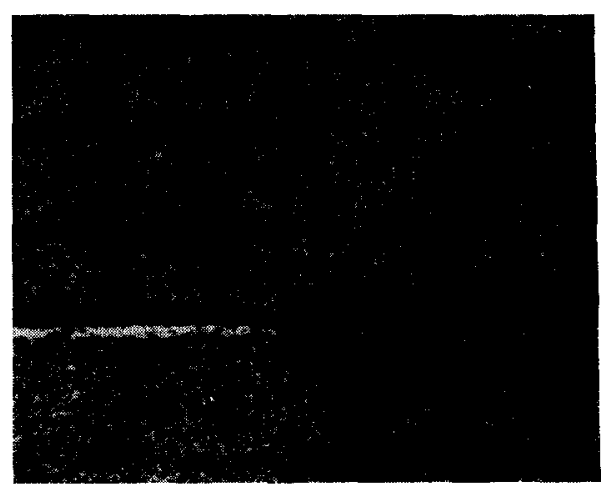

(a)

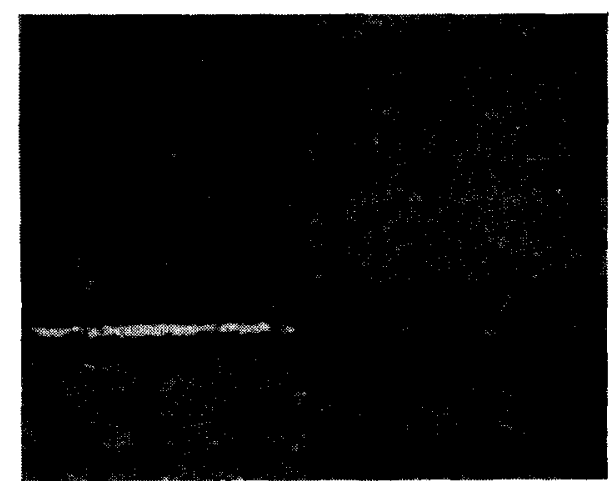

(b)

Figure 7. Photograph of the image with speckling of different samples of graph paper placed together to form the object. (a) Without the amplitude gratings in the illuminating beam, (b) with the vibrating crossed amplitude gratings, projected onto the object.

+In reference [13], page 366, the Fourier transform of $J_{0}$ is given. Since the limit of integrations are finite, a convolution of the last term with the Fourier transform of the exit pupil would have to be carried out. 


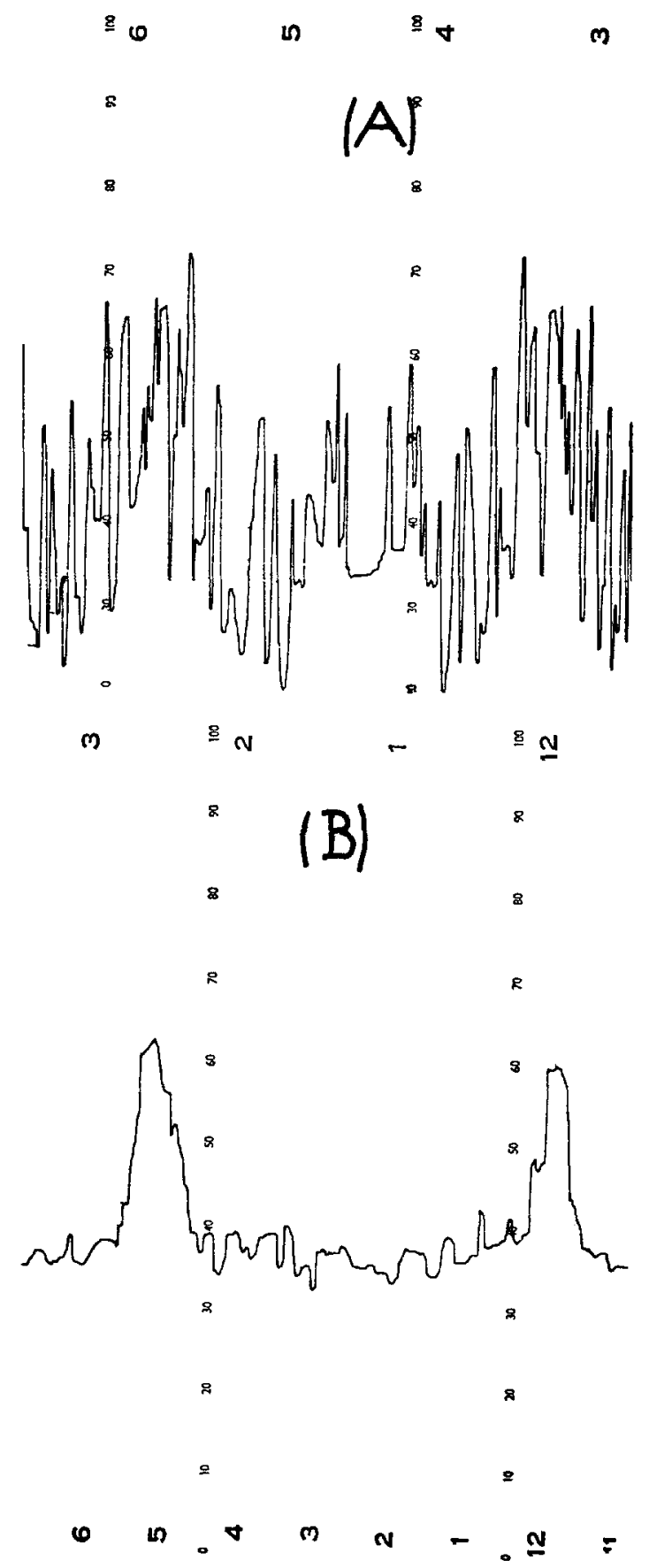

Figure 8. (A) Microdensitometer trace of figure $7(a)$, (B) microdensitometer trace of figure $7(b)$. 
An oscillation or displacement of the object reduces therefore the speckling in the time average except at the neighbourhood of the extreme positions. A plausible explanation of the terms between the square brackets in expression (19) for the oscillating object is the superposition of shifted speckle patterns, where every displacement $\delta u^{\prime}$ contributes an amount weighted by the function

$$
\frac{\operatorname{rect}\left(\delta u^{\prime} / \rho^{\prime}\right)}{\pi \sqrt{ }\left(\rho^{\prime 2}-\delta u^{\prime 2}\right)} .
$$

The speckling disappears in the image in the time average for the oscillating region of the object except in the neighbourhood of $\delta u^{\prime}=\rho^{\prime}$. Furthermore, the contrast of the speckling decreases with increasing amplitude of vibration. The oscillation of the object with fine details on it is not very practical. Instead of oscillating the object we could use an appropriate screen or move the illumination [3]. In an experiment crossed amplitude gratings were projected onto the stationary optically rough object and vibrated normal to its rulings with an amplitude larger than the period. The speckling disappears in the time average for an exposure time longer than the frequency of oscillation. Figure $7(a)$ shows the speckled, magnified image of different samples of graph paper placed together to form the object. With the gratings in vibration, however, the image is seen to be sharp, of good contrast, and free from speckling figure $7(b)$. The frequency of oscillation of the amplitude gratings was 100 c.p.s. and the exposure time half a second. Figure 8 shows the microdensitometer traces of the corresponding part of figures $7(a)$ and $7(b)$ respectively. The circular scanning aperture of $0.03 \mathrm{~mm}$ diameter was chosen to correspond approximately to the size of the speckling. The gratings were used for convenience to prove the analysis, other elements such as lenticular screens or ground glass can be moved to eliminate the speckling in the time average and they need not to be focused onto the object.

\section{ACKNOWLEDGMENTS}

The author would like to thank the Director of the Institute of Technical Physics and the Department of Industrial Research at the ETH-Z, Professor Dr. E. Baumann for encouragement and support of the work. Thanks are further due to other members of the Institute, particularly to Professor W. Baumgartner and W. Witz for critically reading the manuscript and A. Greuter for providing the tuning fork.

Le 'speckling ', phénomène propre à l'éclairage en lumière cohérente, est étudié pour des objets en mouvement latéral. Le speckling est enregistré sur émulsion photographique en exposition double ou moyennée dans le temps. La transformée de Fourier du speckling montre des franges d'interférence correspondant aux mouvements latéraux de l'objet. La méthode proposée livre un outil extrèmement simple pour l'étude de vibrations mécaniques et de mouvements latéraux. Des résultats théoriques et expérimentaux sont exposés.

Die Granulation (speckling), ein Phänomen der kohärenten Beleuchtung, wird für seitlich bewegte Objekte untersucht. Die Granulation wird in der Bildebene auf einer photographischen Emulsion im Zeitmittel und auch zweifach belichtet registriert. Bei der Durchsichtbeleuchtung der entwickelten Photoplatte mit kohärentem Licht ergeben sich in der Fourierebene parallele Interferenzstreifen, deren Abstand und Lage die Bestimmung der Schwingungsamplitude und Richtung ermöglicht. Die Methode ist sehr einfach für die Untersuchungen von mechanischen Schwingungen in der Ebene. Theoretische und experimentelle Resultate werden vorgeführt. 


\section{REFERENCES}

[1] Goldfischer, L. I., 1965, f. opt. Soc. Am., 55, 247.

[2] Hopkins, H. H., and Tiziani, H. J., 1970, Proc. of the Int. Symposium on Holography, Besançon.

[3] Tizian-, H. J., 1967, Thesis, London University.

[4] Archbold, E., et al., 1969, Nature, Lond., 222, 263.

[5] Eliasson, B., and Mottier, F. M., 1971, F. opt. Soc. Am., 61, 559.

[6] Fernelius, N., and Tome, C., 1971, F. opt. Soc. Am., 61, 566.

[7] Leendertz, J. A., 1970, F. Phys. E (Sci. Instrum.), 3, 214.

[8] Burch, J. M., and Tokarski, J. M. J., 1968, Optica Acta, 15, 101.

[9] Fink, W., and Bürger, P. A., 1970, Z. angew. Phys., 30, 176.

[10] ButTers, J. N., and LeEndertz, J. A., 1971, \%. scient. Instrum., 4, 277.

[11] Archbold, E., Burch, J. M., and Ennos, A. E., 1970, Optica Acta, 17, 883.

[12] Hopkins, H. H., 1964, Proc. of the Conference on Photographic and Spectroscopic Optics.

[13] Bracewell, R. M., 1965, The Fourier Transform and its Applications (McGraw-Hill Book Company).

[14] Kozma, A., 1966, J. opt. Soc. Am., 56, 428.

[15] Trziani, H. J., Optik (Proceedings of the DGaO Tagung 1971) (to be published).

[16] KöPF, U., Optik (to be published). 